\title{
LETTER
}

\section{Nonrandom extinction leads to elevated loss of angiosperm evolutionary history}

Jana C. Vamosi ${ }^{1 *}$ and John R. U. Wilson $^{2}$

${ }^{1}$ Department of Biological

Sciences, University of Calgary,

2500 University Drive NW

Calgary, AB T2N 1N4, Canada

${ }^{2}$ Centre for Invasion Biology,

Department of Botany and

Zoology, Stellenbosch

University, Matieland 7600,

South Africa

*Correspondence: E-mail:

jvamosi@ucalgary.ca

\begin{abstract}
The phylogenetic clustering of extinction may jeopardize the existence of entire families and genera, which can result in elevated reductions of evolutionary history $(\mathrm{EH})$, trait diversity, and ecosystem functioning. Analyses of globally threatened birds and mammals suggest current extinction threats will result in a much higher loss of EH than random extinction scenarios, while the analyses of the taxonomical distribution of regionally rare plants find the opposite pattern. The disproportionately high number of rare plant species within species-rich families potentially suggests that lower losses of plant EH will be sustained than expected under random extinction. We show that at a global scale, this is not the case. Species-poor (especially monotypic) angiosperm families are more often at risk of extinction than expected. Because these high-risk species-poor families are as evolutionarily distinct as other families, the expected family-level EH plausibly lost in the next 100 years exceeds that predicted from random extinction by up to $\sim 1165$ million years.
\end{abstract}

\section{Keywords}

Angiosperms, evolutionary history, extinction, IUCN Red List, originality, phylogeny, phylogenetic diversity, rarity, speciation.

Ecology Letters (2008) 11: 1047-1053

\section{INTRODUCTION}

Species extinction not only subtracts a potentially important player from the regional community of interacting organisms, it erases an entry from the history of life. The loss of some species may erase a greater proportion of history due to their long unique evolutionary pathways (Isaac et al. 2007), and potentially result in greater disruption of ecosystem functioning (Maherali \& Klironomos 2007). The phylogeny of organisms is often depicted as a branching tree with the total length from 'trunk' (the ancestor) to all the 'leaves' (the species) representing evolutionary history (EH) (Crozier 1997). Extinction of species thus prunes $\mathrm{EH}$ from the tree. If extinction is random amongst species, most species that go extinct will have surviving close relatives (nearby leaves) and the majority of the total branch length will be conserved (Nee \& May 1997). The extinction of entire clades (e.g. families and genera) harboring large amounts of $\mathrm{EH}$ will be rare (see Fig. 1). Nonrandom extinction has been demonstrated in birds and mammals with the alarming trend that extinction risk is concentrated within smaller genera and families (Purvis et al. 2000), a pattern leading to elevated EH loss. Truly comparable studies within plant lineages have not been attempted. Many localized studies of rarity in plants have found that large families harbor more rare species than do small families (Schwartz \& Simberloff 2001; Lozano \& Schwartz 2005), suggesting that EH could be more easily preserved in plants. It is unclear, however, how much we can, or should, extrapolate patterns of global risk from patterns of local rarity. Considering that over $90 \%$ of the species on the IUCN red list of threatened plant taxa are endemic to a single country (Pitman \& Jorgensen 2002), many threatened plants have not been included in previous datasets (Schwartz \& Simberloff 2001; Webb \& Pitman 2002; Lozano \& Schwartz 2005).

The paucity of comparable global studies on EH loss in plants is likely due in part to the absence of large specieslevel supertrees of plants similar to those in carnivores and primates (Secrest et al. 2002). With upwards to 350000 species of angiosperms projected, an approach that combines taxonomic and phylogenetic information is necessary 
(Purvis et al. 2000; von Euler 2001). With a dated familylevel supertree of angiosperms (Davies et al. 2004) and information on the number of species at-risk in each family from the 2007 IUCN Red List, we compare EH loss predicted from the extinction of observed threatened taxa to that expected under random extinction. If all species from a small family are likely to disappear then we may lose more evolutionary history than if we lose a few species from a large family. Such is clearly the case if we assume that families are roughly the same age. However, the origin of

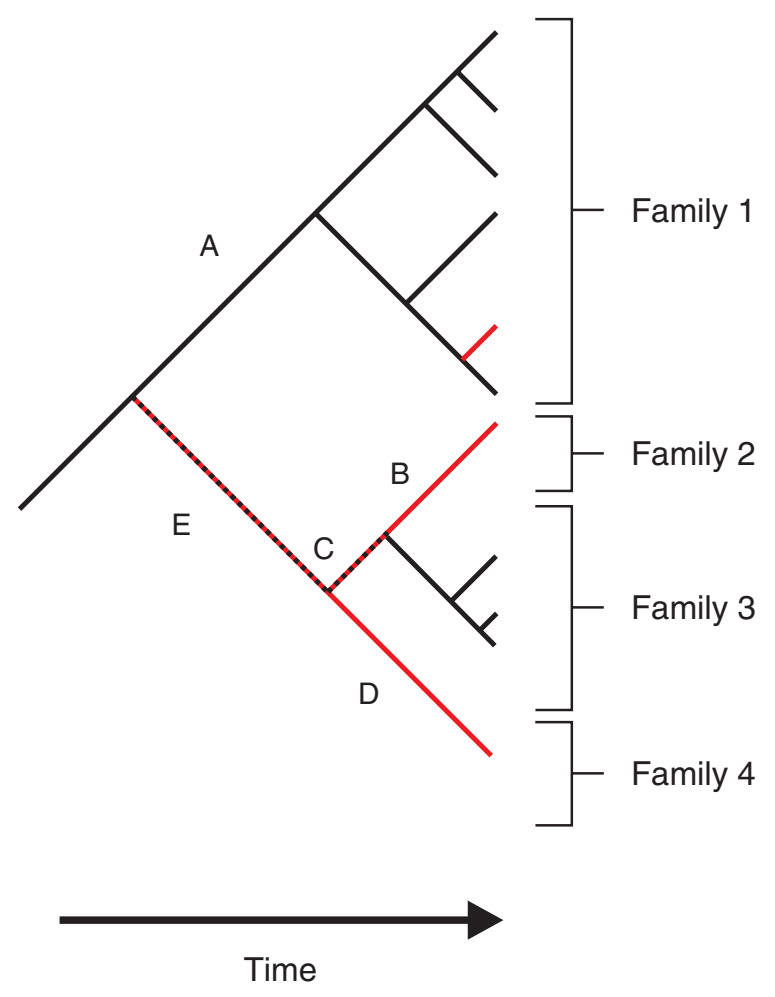

Figure 1 The effects of nonrandom taxonomic and phylogenetic distribution of extinction. If three species belonging to three different families go extinct (red lines), the evolutionary history $(\mathrm{EH})$ and originality $(\mathrm{OG})$ lost depends on the species richness of the family, the age of the family, and the relationship of the family to other at-risk families. For example, extinction of the species in Family 1 poses little risk of losing the $\mathrm{EH}$ along branch A because the family is rich in species (i.e., each species had relatively little OG). Losing the species in Family 2 is of higher consequence because this family is monotypic and we thus lose the $\mathrm{EH}$ along branch B. However, Family 2 is fairly young and its sister family (Family 3) has no at-risk species so the $\mathrm{EH}$ in the $\mathrm{C}$ branch is preserved. Finally, losing the species in Family 3 is of the highest consequence because Family 4 is both monotypic and old (the great amount of EH is lost in branch D). Furthermore, as Family 2 and 4 are closely related, losing both families would place greater importance on the remaining species in Family 3 (they would increase in $O G)$. angiosperm families varies roughly from 4 to 175 mya (Magallon \& Sanderson 2001). If small families have few species simply because they have had limited time to diversify, then losing small families could result in the same amount of evolutionary history lost as losing individual species within older, larger families. Thus, we measure the effect of species extinction on the EH lost with metrics that take into account the species richness of families, the age of families, and the evolutionary distance of extinction-prone families to other families (see Fig. 1).

We also use a complementary metric, originality (OG), that apportions the $\mathrm{EH}$ in the entire phylogeny to each of the terminal nodes in the tree based on how related that node is to all others. Originality metrics provide a composite value of the $\mathrm{EH}$ within a family (the terminal branch) and how unique that $\mathrm{EH}$ is in the entire phylogeny (i.e., how many other families share common $\mathrm{EH}$ with a particular family deeper in the tree). Like EH, a species belonging to an old family has, all else being equal, higher OG than a species belonging to a young family (Fig. 1). Unlike with $\mathrm{EH}$, however, a pair of early-diverging sisterfamilies will maintain high OG, even if they diverged from each other rather recently. Species in families with higher OG arguably warrant additional conservation attention [e.g. may be granted high priority status as Evolutionary Distinct Globally Threatened (EDGE) species (Isaac et al. 2007)].

It is important to note that only a proportion of all at-risk species will actually go extinct and a small proportion of non-threatened species will go extinct before they are listed. These extinction probabilities greatly influence whether we predict entire families to go extinct. Here, we assume these extinction probabilities within a family are independent, thus greatly improving the chances that at least one species within mid- to large families will escape extinction and the family-level $\mathrm{EH}$ and OG will be preserved. The probability of extinction of threatened species depends to some degree upon human intervention to prevent the extinction of these species, although many other factors such as life history and breeding systems may be involved (Vamosi \& Vamosi 2005a). The degree to which these extinction probabilities alter the predicted EH or OG loss may help determine the importance of future conservation intervention.

\section{MATERIALS AND METHODS}

The number of threatened species per family was the sum of species having the IUCN threat categories (v3.1) of Vulnerable (VU), Endangered (EN), and Critically Endangered (CR) according to the 2007 IUCN Red List. Least Concern (LC) and Near Threatened (NT) were considered 
'not threatened' (nt), as were species that have yet to be evaluated. These IUCN threat categories have specific criteria based on reductions in range and population size and should be relatively immune to taxonomic biases (see IUCN 2001). Because only a portion of the family represented in the 1997 Red List have had their status re-classified according to new guidelines of the 2007 Red List, the threat status of species listed in the 1997 and 2007 versions were combined (Vamosi \& Vamosi 2005a). When a family was listed in both versions, we recorded the number of threatened species that was higher, yet likely these values still represent underestimates in many cases. The family memberships of threatened species were adjusted to conform to the taxonomy of the 439 families recognized by the Angiosperm Phylogeny Group (APGII 2003), updated where necessary with information from the Angiosperm Phylogeny Website (Stevens 2001 onwards). The total number of species per family followed Mabberley (1997).

\section{Evolutionary history at risk}

The combined EH of all the angiosperm orders and families was estimated at 35244 million years by summing the ages of the separate clades over the angiosperm phylogeny. The dated phylogeny used for this was that presented by (Davies et al. 2004), updated with information from the Angiosperm Phylogeny Website (Stevens 2001 onwards) where data were not available. We summed the EH and OG (calculated as quadratic entropy from Pavoine et al. (2005)) for all extinct families. This effectively assumes that all threatened species go extinct simultaneously. For the OG metric, however, the extinction of any particular species or family alters the OG, or evolutionary distinctiveness, of all the remaining species. Because we have little information on the sequence in which the current threatened species will go extinct, we cannot explore this in more detail but it may warrant more investigation in future.

The probability of extinction of an entire family with consequent loss of its EH and OG (i.e., the 'trunk' EH or OG) occurs when all species of the family go extinct (Fig. 1). Assuming that extinction events within a family are independent of one another, the probability of extinction of an entire family and the consequent loss of its EH and OG can be calculated as:

$$
\left(\begin{array}{l}
\mathrm{N} \\
\mathrm{R}
\end{array}\right) p^{R} b^{N-R}
$$

where $R$ is the number of red-listed species in a family with $N$ species, $p$ is the probability that a red-listed species goes extinct and $b$ is the low, but non-zero, probability that a species that is not at-risk goes extinct in the next 100 years.
We examine the effects of taxonomic/phylogenetic bias in extinction rates over the full spectrum of theoretical extinction probabilities of threatened species $(0 \leq p \leq 1)$ while the chance of non-threatened (nt) species going extinct (background extinction, or $b$ ) is kept constant at $1 \%$. Based on the number, and probability of extinction in the next 100 years, on the 2007 Red List - CR (99\% chance of extinction in the next 100 years), EN (33\% chance of extinction in the next 100 years), and VU (10\% chance of extinction in 100 years (Redding \& Mooers 2006)) - a weighted mean 'ballpark' probability of extinction for all threatened species can be roughly estimated to be $32 \%$ in the next 100 years. More accurate estimates of risk are unachievable at this time because a great many angiosperm species have not been reanalyzed since 1997 to conform to 2001 categories (i.e., we only know that species were either CR, VU, or EN as of 1997). Eq. 1 highlights the effect of species richness on family-level extinction, independent of extinction risk designations (a non-threatened monotypic family has a 0.01 chance of going extinct randomly within the next 100 years - the same probability of a family with four species, all of which are threatened $\left(0.32^{4} \approx 0.01\right)$ ). Because most families have many species, the loss of the trunk EH of most families is very close to zero, making this analysis robust to the assumption that species that are "Not Evaluated' are not threatened (most unevaluated species belong to larger families).

There are 16498 angiosperm species presently at risk (or $7.48 \%$ of the 246317 described species). We calculated the number of species expected to be at risk in each family under a random binomial distribution in 10000 randomizations [generated using $\mathrm{R}$ version 2.6.0 ( $\mathrm{R}$ Development Team 2007)] assuming every species has a $7.48 \%$ chance of being at risk. This randomization approach (see eqn 1) avoids the pitfalls associated with the statistical analysis of proportions superimposed upon species richness variation (i.e., monotypic families have either $0 \%$ or $100 \%$ of their species at-risk whereas this is rarely the case for large families).

We then ask whether more families, EH, or OG was predicted to go extinct compared to the distribution of these variables under conditions of random extinction. We set $b$ to 0.01 and examined $p$ between the values of 0 and 1 . We further investigated the effect of the phylogenetic distribution of risk by examining whether (1) the families currently at risk of extinction have more or less $\mathrm{EH}$ or OG than families not at-risk, (2) whether, for a given number of family extinctions, the current at-risk families harbor more or less EH and OG than randomly sampled families, and (3) additional $\mathrm{EH}$ is lost because at-risk families are often closely-related, leading to a loss of the $\mathrm{EH}$ inherent in the common branch between the families (Heard \& Mooers 2000) (see Fig. 1). To estimate the likelihood of this third possibility, we used Phylocom (Webb et al. 2004) to detect a 
phylogenetic signal in the logit-transformed proportions of species at-risk (Purvis et al. 2000) and in the calculated probabilities of family extinction. We also conducted a nonnested sister-group analysis of the risk per family between smaller and larger clades, comparing whether clades with similar evolutionary history show differences in risk that correlate with species richness differences (Vamosi \& Vamosi 2005b).

\section{RESULTS}

Whereas $\sim 7.5 \%$ of all angiosperm species are listed as at risk on the IUCN Red List, averaged at the family level 11\% of species per family are at risk. If the extinction of each species within a family is an independent event, the average estimated rate of family-level extinction within the next 100 years is relatively low (mean risk of family extinction is $0.6 \%$ ) because most families have many species. However, $37 \%$ (19 out of 52) of monotypic angiosperm families are at risk (CR, EN, or VU). Family-level extinction will be concentrated within these 19 families (sensu Angiosperm Phylogeny Group 2003), namely Aphloiaceae, Aphyllanthaceae, Bretschneideraceae, Cephalotaceae, Desfontainiaceae, Drosophyllaceae, Gomortegaceae, Hesperocallidaceae, Ixerbaceae, Koeberliniaceae, Lactoridaceae, Medusagynaceae, Pentadiplandraceae, Plocospermataceae, Rhoipteleaceae, Rhynchocalycaceae, Setchellanthaceae, Tetracarpaeaceae, and Ticodendraceae. There are also three ditypic families with every member at risk (Aphanopetalaceae, Dirachmaceae and Medusandraceae) for a total of 22 at-risk families (families where $100 \%$ of the species are threatened). All other families, despite potentially having high proportions of at-risk species, are predicted to have surviving species within the next 100 years, resulting in a low probability of extinction for the family as a whole.

This clustering of extinction risk in species-poor families means that there are far more at-risk families than expected by chance ( 22 vs. 4 ; see Fig. 2 ). The estimated number of these families that we will realistically lose depends on our projections of extinction of at-risk species (see Methods), which in turn depend on our efforts to conserve at-risk species. Within the near future (the next 100 years), it is estimated that roughly one-third of at-risk species will actually be lost (see Methods); we can therefore expect seven families to go extinct (cf. 2 under random species extinction; Fig. 2). Re-analysis of the data using only families recognized under the Brummitt (1992) system of classification produced similar results, suggesting that the findings are robust to taxonomic treatment.

The EH of each family, and the proportion of $\mathrm{EH}$ that is unique to each family [as calculated with quadratic entropy (Pavoine et al. 2005)] are summarized in the online Appendix. The EH and OG lost is roughly proportional

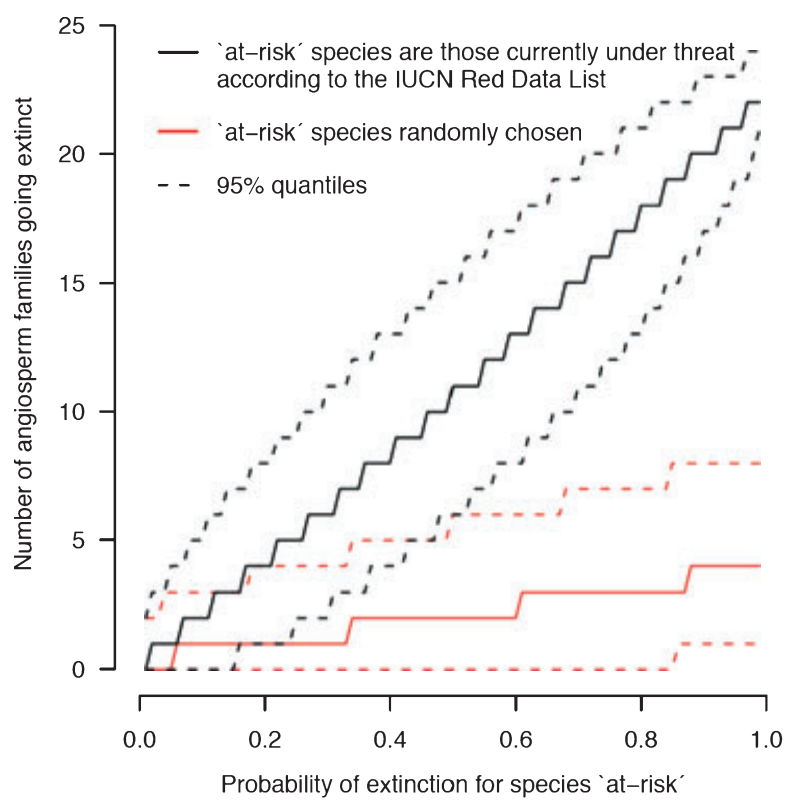

(the probability of extinction for species not 'at-risk' is always 0.1 )

Figure 2 More angiosperm families will go extinct if currently threatened species are lost than if random species are lost. Examination of the difference between the black and red lines when $100 \%$ of the threatened species go extinct conveys the degree to which threat is concentrated in species-poor families; 18 more families have every member threatened compared to random expectations (lost families are often monotypic). Including more realistic extinction projections (e.g. $\sim 32 \%$ of threatened species will go extinct rather than $100 \%$ ), we still expect to lose significantly more families than expected under random extinction scenarios.

to the number of families lost in both scenarios of extinction (see Suppl Fig. S1). This is because monotypic and non-monotypic families harbor equivalent $\mathrm{EH}$ (69.4 vs. 71.9 million years ago; $\left.F_{1437}=0.43, P=0.51\right)$ and originality (OG, $\left.F_{1437}=0.009 ; P=0.92\right)$ Grouping the 22 at-risk families (monotypic + ditypic) together reveals that at-risk families have EH or OG equivalent to families that are not at high risk of extinction (EH: $F_{1437}=1.48$; $P=0.22$; and OG: $F_{1437}=1.56 ; P=0.21$, and see Suppl Fig. S1). Therefore, extinction risk is not clustered in particularly evolutionarily distinct angiosperm families. In fact the EH and OG lost per family is slightly lower for threatened families than a random selection (Fig. S1).

Given the threat to species-poor families, in the worstcase scenario of losing all 'at-risk' families, $4.1 \%$ of the estimated 35244 million years of angiosperm family evolutionary history (or 1432 million years) will be lost vs. the 266 million years $(0.75 \%)$ expected through chance (Fig. 3a), resulting in an additional $\sim 1165$ million years of angiosperm evolutionary history at risk. Originality showed similar but more variable results (Fig. 3b). This is because 

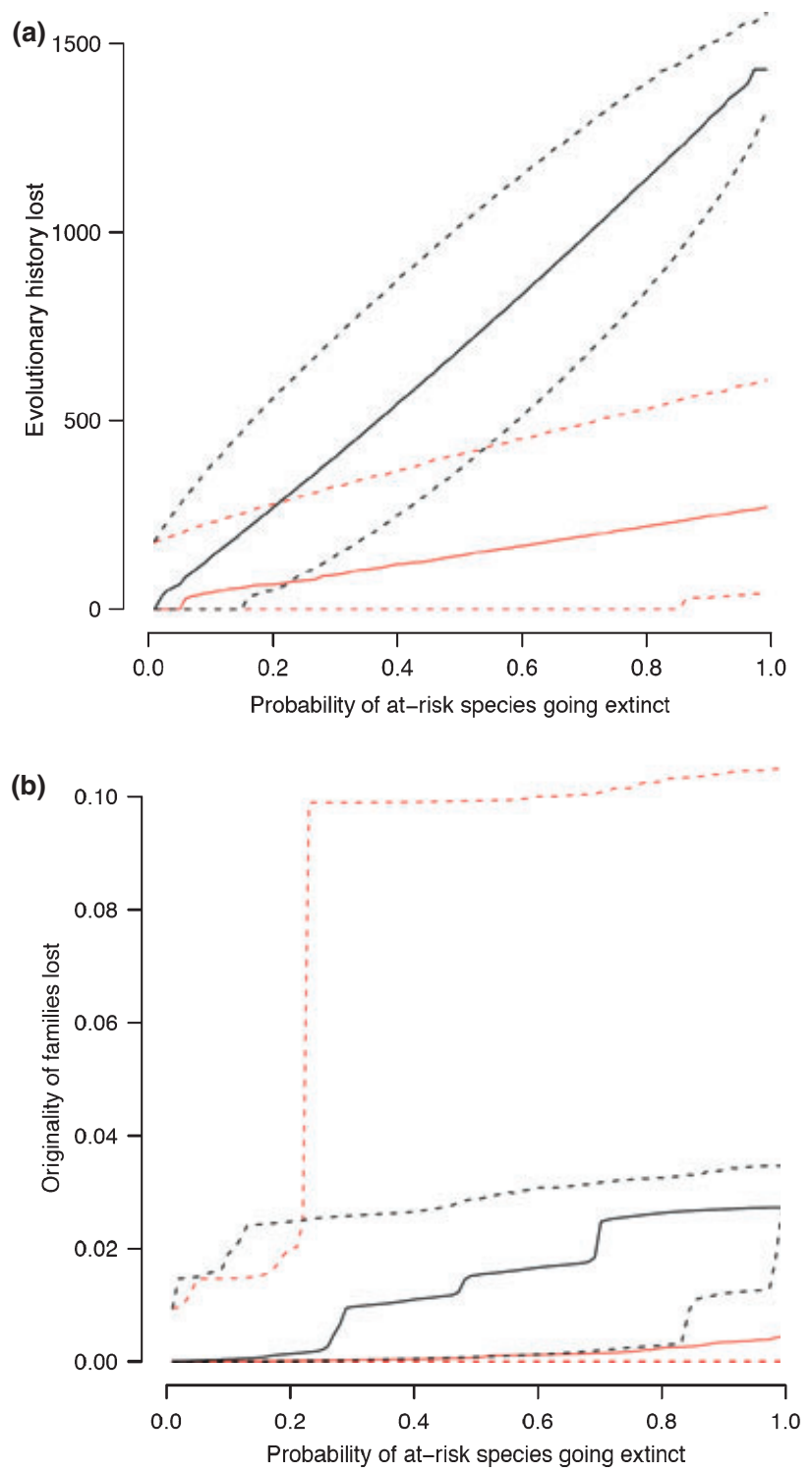

Figure 3 The expected loss of (a) evolutionary history, EH, and (b) originality (OG) due to predicted family extinction compared to random simulations (10 000 randomizations at 0.01 intervals of $P(b=0.01$ for all). Because the threatened families do not contain less $\mathrm{EH}$ than random families (i.e., they are not particularly 'old' or 'new' families; see Fig. S1), additional EH loss and OG is predicted. However, the difference between real and random extinction is higher for $\mathrm{EH}$ than OG because many evolutionarily distinct clades (e.g., families on basal branches related to few other families) are not presently threatened. In random simulations, when these families are chosen for extinction they result in a great loss of OG but, unless their sister-family is also randomly chosen, not a great loss in $\mathrm{EH}$.

originality heavily upweights the value of families with few closely related clades. There are several species-poor families on basal lineages in the angiosperms that have no currently threatened species; yet future assessments may change this outcome (in Amborellaceae, Austrobaileyaceae, and Cabombaceae in particular). As these families have a high contribution to overall originality, their occasional extinction in randomizations result in large variance in originality lost under random extinction. Thus, the originality lost under random extinction depends heavily on the phylogenetic placement of the randomly targeted species and the degree of tree imbalance. Indeed, this highlights the need to conserve species-poor but evolutionary-distinct families regardless of how many of their species are at risk.

How much of the additional EH history loss can be attributed to related families being at-risk? The above analysis incorporates the fact that families are not independent entities, by calculating the evolutionary history when related species or families go extinct but does not explicitly measure the extent to which extinction risk is phylogenetically conserved. Rates of risk were observed to have a strong phylogenetic signal $(P=0.002)$, indicating that sisterfamilies are more likely to experience similar levels of risk [perhaps because of common traits (Pilgrim et al. 2004; Kotiaho et al. 2005; Vamosi \& Vamosi 2005a), common geographical ranges, or ecological interactions (Rezende et al. 2007)]. However, this in itself is unlikely to contribute much to increased loss of evolutionary history because of widespread and frequent shifts in diversification rates in the angiosperm phylogeny (Davies et al. 2004). Thus, because a small family is unlikely to have a small sister-group, the probabilities of the entire family going extinct exhibited no phylogenetic signal $(P=0.338)$. Furthermore, non-nested sister-group analysis of families at the tips of the phylogeny, revealed that the odds of being threatened is higher within smaller families than it is within their larger sister-clades ( $P=0.02$; Wilcoxon signed rank test), indicating that the reason small families have fewer species may be because they have been experiencing elevated extinction risk over evolutionary time scales just as they do in the present day (Vamosi \& Vamosi 2005a).

\section{DISCUSSION}

Much evolutionary history $(\mathrm{EH})$ can be preserved in the face of substantial species extinction if extinction risk is random with respect to the phylogenetic position of species (Nee \& May 1997). A less optimistic forecast for the current extinction crisis can arise when extinction is nonrandom, however, because the mean EH loss per species accelerates when there is a negative relationship between diversification rates and extinction risk (Heard \& Mooers 2000). One of the major conclusions of this study is that extinction risk of angiosperms is not randomly distributed but clustered within taxa that have few species; a result forecasting elevated EH loss compared to random simulations. This 
pattern mirrors recent findings in animal lineages studied thus far (Purvis et al. 2000) with the somewhat heartening exception that, unlike the patterns found in birds (Gaston \& Blackburn 1997) and marsupials (Johnson et al. 2002), threatened angiosperm families are not particularly old nor evolutionary distinct. However, our findings are at odds with those of many regional studies of plant rarity (Schwartz \& Simberloff 2001; Webb \& Pitman 2002; Lozano \& Schwartz 2005), which have found rarity disproportionately high within species-rich families. The disparity in results with most regional rarity studies is likely caused by the inclusion of data on more small families with limited global distribution (e.g., Dirachmaceae on Socotra, Barbeyaceae in NE Africa, and Cardiopteridaceae in SE Asia), and the fact that rarity designations used in regional studies are very different from the globally threatened status used by the IUCN Red List. As others before it (Brook et al. 2006), this study highlights the pitfalls of extrapolating extinction risk from rarity.

The EH lost under predicted extinction relative to random extinction increases as the overall extinction risk increases. This is due to the extreme tree imbalance seen in the angiosperm phylogeny (Davies et al. 2004). As additional species go extinct in random simulations, the vast majority of them are in large families, leading to little $\mathrm{EH}$ loss. Effectively, calculating EH and OG loss in this way assumes that all within-family phylogenies have the general tree shape of a long common branch (or 'trunk') with a burst of rapid diversification in the recent past. Thus, each species contributes a negligible amount of $\mathrm{EH}$ on top of the familylevel EH. Although recent studies suggest this may be an appropriate assumption for many plant lineages (e.g. Cowling \& Pressey 2001), we await species-level supertrees of angiosperms to provide a more complete picture of estimates of $\mathrm{EH}$ loss within and between families.

With prudent adjudication of conservation resources, we may be able to circumvent elevated EH loss by prioritizing species in small families, as is currently advocated by researchers of amphibians and mammals (Isaac et al. 2007). On the other hand, small families are at high risk even when compared to their sister-groups, indicating that parallels may exist between traits that have kept a family relatively speciespoor historically (perhaps via increased extinction) and traits that presently confer high extinction risk. This possibility raises the questions of whether (1) high-risk species would be at a higher risk of extinction even without the contribution from human-induced disturbance or (2) have limited evolutionary potential to contribute to future biodiversity. Nevertheless, the loss of higher taxa and their unique traits could translate to a loss of functional diversity and a decrease in ecosystem function (Maherali \& Klironomos 2007). Future investigation of these possibilities is necessary and will no doubt fuel controversy on the importance of preferentially conserving evolutionary distinct species.

\section{ACKNOWLEDGEMENTS}

We thank Ş. Procheş, S. Vamosi, A. Mooers, and the inspiring crew of FAB-lab at SFU for comments and helpful advice on the manuscript. This project was funded by the Natural Sciences and Engineering Research Council of Canada (JCV) and the DST-NRF Centre of Excellence for Invasion Biology (JRUW).

\section{REFERENCES}

Angiosperm Phylogeny Group (2003). An update of the Angiosperm Phylogeny Group classification for the orders and families of flowering plants: APG II. Bot. J. Linn. Soc., 141, 399-436.

Brook, B.W., Traill, L.W. \& Bradshaw, G.E. (2006). Minimum viable population sizes and global extinction risks are unrelated. Ecol. Lett., 9, 375-382.

Brummitt, R.K. (1992). Vascular Plant Families and Genera. Royal Botanic Gardens, Kew.

Cowling, R.M. \& Pressey, R.L. (2001). Rapid plant diversification: planning for an evolutionary future. Proc. Nat. Acad. Sci. USA, 98, 5452-5457.

Crozier, R.H. (1997). Preserving the information content of species: genetic diversity, phylogeny, and conservation worth. Ann. Rev. Ecol. Syst., 28, 243-268.

Davies, T.J., Barraclough, T.G., Chase, M.W., Soltis, P.S., Soltis, D.E. \& Savolainen, V. (2004). Darwin's abominable mystery: insights from a supertree of the angiosperms. Proc. Nat. Acad. Sci. USA, 101, 1904-1909.

von Euler, F. (2001). Selective extinction and rapid loss of evolutionary history in the bird fauna. Proc. Roy. Soc. Lond. B, 268, $127-$ 130.

Gaston, K.G. \& Blackburn, T.M. (1997). Evolutionary age and the risk of extinction in the global avifauna. Evol. Ecol., 11, 557-565.

Heard, S.B. \& Mooers, A.Ø. (2000). Phylogenetically patterned speciation rates and extinction rates change the loss of evolutionary history during extinctions. Proc. Roy. Soc. Lond. B, 267, 613-620.

Isaac, N.J.B., Turvey, S.T., Collen, B., Waterman, C. \& Baillie, J.E.M. (2007). Mammals on the EDGE: conservation priorities based on threat and phylogeny. PLOS ONE, 3, e296.

IUCN (2001). IUCN Red List Categories and Criteria: Version 3.1. IUCN, Gland, Switzerland and Cambridge, UK.

Johnson, C.N., Delean, J. \& Balmford, A. (2002). Phylogeny and the selectivity of extinction in Australian marupials. Anim. Cons., 5, 135-142.

Kotiaho, J.S., Kaitala, V., Komonen, A. \& Paivinen, J. (2005). Predicting the risk of extinction from shared ecological characteristics. Proc. Nat. Acad. Sci. USA, 102, 1963-1967.

Lozano, F.D. \& Schwartz, M.W. (2005). Patterns of rarity and taxonomic group size in plants. Biol. Cons., 126, 146-154.

Mabberley, D.J. (1997). The Plant Book: A Portable Dictionary of the Higher Plants. Cambridge University Press, Cambridge.

Magallon, S. \& Sanderson, M.J. (2001). Absolute diversification rates in angiosperm clades. Evolution, 55, 1762-1780. 
Maherali, H. \& Klironomos, J.N. (2007). Influence of phylogeny on fungal community assembly and ecosystem functioning. Science, 316, 1746-1748.

Nee, S. \& May, R.M. (1997). Extinction and the loss of evolutionary history. Science, 278, 692-694.

Pavoine, S., Ollier, S. \& Dufour, A.-B. (2005). Is the originality of a species measurable? Ecol. Lett., 8, 579-586.

Pilgrim, E.S., Crawley, M.J. \& Dolphin, K. (2004). Patterns of rarity in the native British flora. Biol. Cons., 120, 161-170.

Pitman, N.C.A. \& Jorgensen, P.M. (2002). Estimating the size of the world's threatened flora. Science, 298, 989.

Purvis, A., Agapow, P.-M., Gittleman, J.L. \& Mace, G.M. (2000). Nonrandom extinction and the loss of evolutionary history. Science, 288, 328-330.

R Development Team (2007). R: A Language and Environment for Statistical Computing. R Foundation for Statistical Computing, Vienna.

Redding, D.W. \& Mooers, A.Ø. (2006). Incorporating evolutionary measures into conservation prioritization. Cons. Biol., 20, 16701678.

Rezende, E.L., Lavabre, J.E., Guimaraes, P.R., Jordano, P. \& Bascompte, J. (2007). Non-random coextinctions in phylogenetically structured mutualistic networks. Nature, 448, 925-928.

Schwartz, M.W. \& Simberloff, D. (2001). Taxon size predicts rates of rarity in vascular plants. Ecol. Lett., 4, 464-469.

Secrest, W., Brooks, T.M., da Fonseca, G.A.B., Konstant, W.R., Mittermeier, R.A., Purvis, A. et al. (2002). Hotspots and the conservation of evolutionary history. Proc. Nat. Acad. Sci. USA, 99, 2067-2071.

Stevens, P.F. (2001 onwards). Angiosperm Phylogeny Website. Version 8, June 2007. http://www.mobot.org/MOBOT/research/APweb/.

Vamosi, J.C. \& Vamosi, S.M. (2005a). Present day risk of extinction may exacerbate the lower species richness of dioecious clades. Divers. Distrib., 11, 25-32.

Vamosi, S.M. \& Vamosi, J.C. (2005b). Endless tests: guidelines to analyzing non-nested sister-group comparisons. Evol. Ecol. Res., $7,567-579$.
Webb, C.O. \& Pitman, N.C.A. (2002). Phylogenetic balance and ecological evenness. Syst. Biol., 51, 898-907.

Webb, C.O., Ackerly, D. \& Kembel, S. (2004). Software for the Analysis of Community Phylogenetic Structure and Character Evolution. Version 3.22. (http://www.phylodiversity.net/phylocom).

\section{SUPPORTING INFORMATION}

Additional Supporting Information may be found in the online version of this article.

Appendix S1 Families recognized by the Angiosperm Phylogeny Group (APGII 2003), total number of species, total number of threatened species, evolutionary history $(\mathrm{EH})$ and originality [OG; as calculated in Pavoine et al. (2005)] used in the analysis.

Figure S1 Loss of threatened families (black lines) do not result in more or less (a) EH or (b) originality lost per family than the loss of random families (i.e. they are not particularly 'old' or 'new' families).

Please note: Blackwell Publishing are not responsible for the content or functionality of any supporting materials supplied by the authors. Any queries (other than missing material) should be directed to the corresponding author for the article.

Editor, Ross Crozier

Manuscript received 10 April 2008

First decision made 15 May 2008

Manuscript accepted 28 May 2008 